\title{
Postural Adaptations To Supra-postural Tasks in Children With Developmental Coordination Disorder
}

\author{
Fu-Chen Chen", Thomas A Stoffregen ${ }^{*} \&$ Michael G. Wade \\ (*)University of Minnesota, USA \\ E-mail: chen1619@umn.edu,tas@umn.edu,mwade@umn.edu
}

\begin{abstract}
We investigated the effect of varying memory (cognitive) demands, and visual (perceptual) demands on postural motion. Sixty four children (32 DCD, 32 TDC, 9-to-10 years) were volunteers. Each performed separate memory and visual tasks at two levels of difficulty; easy (LD) and hard (HD) while recording their postural motion.

For the memory task, both groups reduced postural sway in the HD condition. For the visual task only the TDC group reduced postural sway in the HD condition; DCD children did not. The DCD group did not reduce postural motion but, in fact, increased motion. We also found several group $\times$ task interactions on sway. Our data suggest a weakening of the action linkage between both cognitive and perceptual tasks in children diagnosed with movement difficulties. The data are discussed in the context of limitations in the embodied relationship between posture and both perceptual and cognitive activity.
\end{abstract}

\section{Introduction}

The term Developmental coordination disorder (DCD) refers to developmental impairments of motor coordination and difficulties in movement skills that children exhibit, that are not derived from general intellectual, sensory or motor neurological impairment [1]. The prevalence of DCD is highest among elementary school-aged children, where the problem is often disregarded, and as a consequence children with DCD are stigmatized as "clumsy", " physically awkward" or worse "lazy".

In children with DCD, motor skill ability is below that of age-matched typically developing children (TDC). Children with DCD demonstrate a wide spectrum of motor coordination difficulties that include unstable stance, awkward running patterns, poor handwriting, drawing, and scissoring. Previous research has reported group differences in the control of postural motion while standing on a force plate $[2,3$, 4] or in a swinging room (Wann, Mon-Williams, \& Rushton,1998) when comparing children with and without a DCD diagnosis. More importantly, Geuze [2] and Tsai et al. [4] found that the differences in postural motion between the two groups was more noticeable in more challenging conditions (e.g. eyes closed v.s. open, and one-leg vs. two-leg stance). Geuze and Borger suggested that group differences in such measures may be exacerbated in dual task protocols [5].

In healthy adults and typically developing children, the magnitude of standing body sway is often modulated by variations in the ocular demand of suprapostural visual tasks. Stoffregen, Riley,Hove, Bonnet, and Bardy [6] compared body sway in healthy adults during performance of a cognitive task (mental arithmetic) and a visual perceptual task (signal detection) that were matched for subjective mental workload. They found that body sway was greater during mental arithmetic than during signal detection. By contrast, sway was not affected by variations in the difficulty of purely cognitive tasks (easy vs. hard mental arithmetic). Chang, Wade, Stoffregen, Hsu, and Pan [7] compared sway in children with and without autism spectrum disorder (ASD). Children with ASD tended to sway more than typically developing children; however, both groups reduced their sway during performance of a demanding visual task, relative to sway during a less demanding visual task.

The present study focused on postural responses of children with and without DCD while engaged in supra-postural tasks. To date studies using postural and supra-postural dual-task protocols are limited. We investigated the postural motion responses among children with and without DCD while they engaged in a signal detection and a digit memory task.

\section{Method}

\section{Participants}

This is an Open Access article distributed under the terms of the Creative Commons Attribution-Noncommercial License 3.0, which permits unrestricted use, distribution, and reproduction in any noncommercial medium, provided the original work is properly cited. 
Sixty-two volunteer children (32 DCD and 32 TDC) from a Primary School in Kaohsiung, Taiwan. Each was of normal intelligence and free from a diagnosis of attention deficit and hyperactivity disorder.

\section{Apparatus}

Postural motion data for both head and torso were recorded using a magnetic tracking system (FasTrak, Polhemus Inc., Colchester VT, USA).

\section{Signal detection and digit memory tasks}

Each participant performed both the digit memory task and signal detection tasks during stance (Figure 1). A signal detection task comprised pairs of vertical lines generated by a custom application presented on a 14.1 inch laptop screen placed 1 meter from the participant. Each pair of vertical lines were parallel to each other, apart horizontally by $1.09^{\circ}$ of visual angle, displayed against a white background. In the LD condition (Figure 2, upper part), the neutral events were two vertical lines identical in length with $1.26^{\circ}$ of vertical extent, and critical signals were two vertical lines different in length with $1.26^{\circ}$ and $1.50^{\circ}$ of vertical extent for left and right lines separately while the contrast ratio between black lines and white background was approximately $1: 26$. In the HD condition (Figure 2, lower part), the neutral event were two vertical line in the same length with $1.26^{\circ}$ of vertical extent and critical signals were comprised of two different length vertical lines with $1.26^{\circ}$ and $1.38^{\circ}$ of vertical extent for left and right lines separately while the contrast ratio between grey lines and white background was approximately $1: 1$. Presentation of the visual stimuli (pairs of lines) lasted $444.44 \mathrm{~ms}$, with a inter-stimulus interval (showing a blank white screen) lasting $888.88 \mathrm{~ms}$ A total of 90 signals, 60 neutral and 30 critical signals comprised each 120second trial. Participants held a computer mouse and were asked to click it in response to a critical signal while postural motion was continuously recorded.

Participants also performed a digit memory task [8]. Participants were pre-tested to determine an individual 's maximum number of digits each was able to recall correctly in 10 -seconds. This maximum number served as the HD condition. The LD condition was $50 \%$ of the HD number. If the maximum number (HD) was odd, the number was rounded down for the LD condition. The random number digit spans were created with Microsoft Excel. Each digit string was presented for 10 seconds, on a 14.1 inch laptop screen placed one meter in front of each participant. Participants were asked to remember and mentally rehearse the digit string during quiet stance until the digits disappeared and screen went white. At that moment, the 120-seconds postural motion recording commence; participants continued to mentally rehearse the previously viewed digit string. Participants were asked to recall the digit string after the 120 -seconds period.

\section{Procedure}

The study was conducted in a sound-proof room. Postural motion was collected under two conditions of task difficulty, LD and HD. Participants completed 3 trials each for the LD and HD condition. Trials were blocked by task difficulty conditions, and block order was random and counterbalanced across participants. Half of the children performed the LD condition first, and the other half the HD condition first.
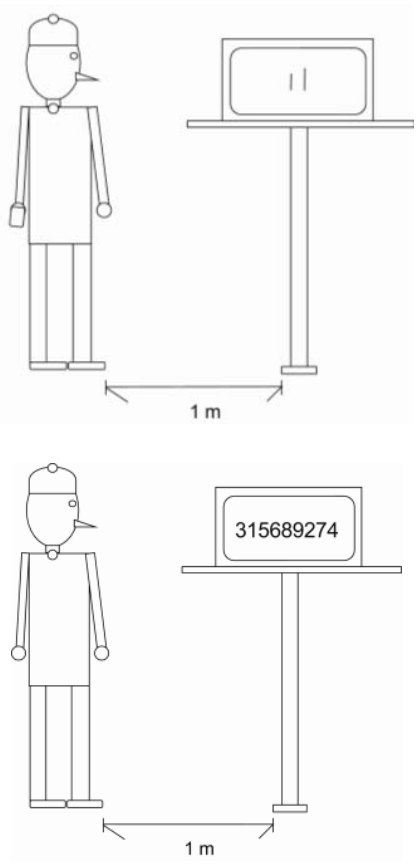

Figure1. Experimental setting for (upper) signal detection task, and (lower) digit memory task. 


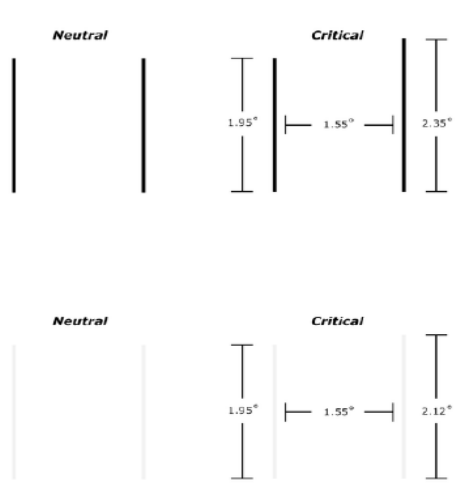

Figure 2. Signal detection task: : low difficulty (upper ) and high difficulty (lower ) conditions.

\section{Procedure}

For postural motion data, we evaluated the positional variability (standard deviation of position), with separate analyses for movement in the AP (anterior-posterior) and ML (medio-lateral) axes for head and torso.

\section{Results}

In signal detection task, a significant Group $\times$ Task Difficulty interaction was significant for positional variability in the AP axis for the head, $F(1,62)=5.15$, $p<.05$, and torso, $F(1,62)=5.89, p<.05$; and for positional variability for the torso in the ML axis, $F(1$, $62)=4.59, p<.05$. The interaction effects revealed that the effect of visual task (HD vs. LD) on postural activity differed quantitatively between the TDC and DCD groups. Figure 3 illustrates that while the TDC group reduced postural motion in the HD relative to the LD condition, the DCD group actually increased their postural motion.

For the digit memory task, the Task Difficulty $\times$ Group interaction was significant for ML movement of the head, $\mathrm{F}(1,62)=5.56, \mathrm{p}<.05$, and of the torso, $\mathrm{F}$ $(1,62)=4.72, p<.05$. Figure 4 illustrates that reduction of postural motion was greater for the TDC group than the DCD group: The TDC group modulated their postural motion as a function task difficulty, but the DCD group did not. (Figure 4).
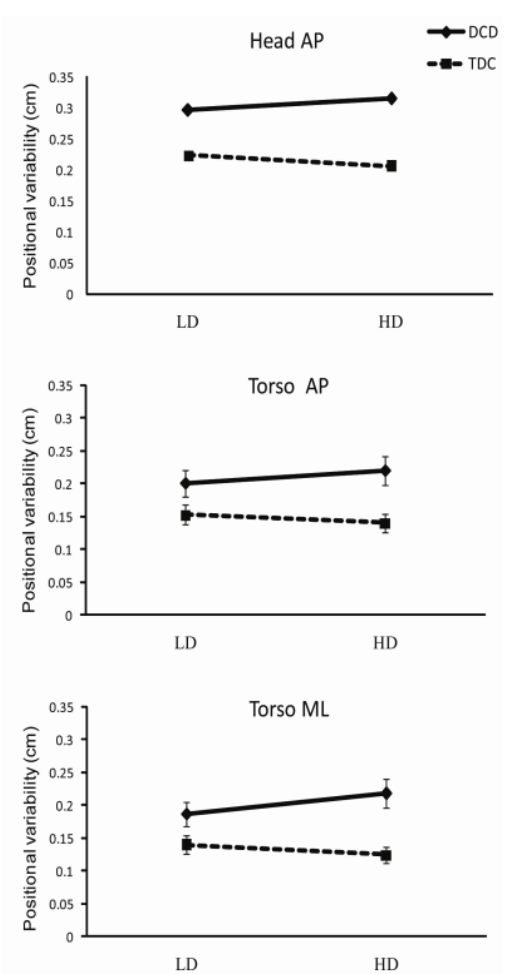

Figure 3.Positional variability of head in the AP axis, and of torso in the AP and ML axes.
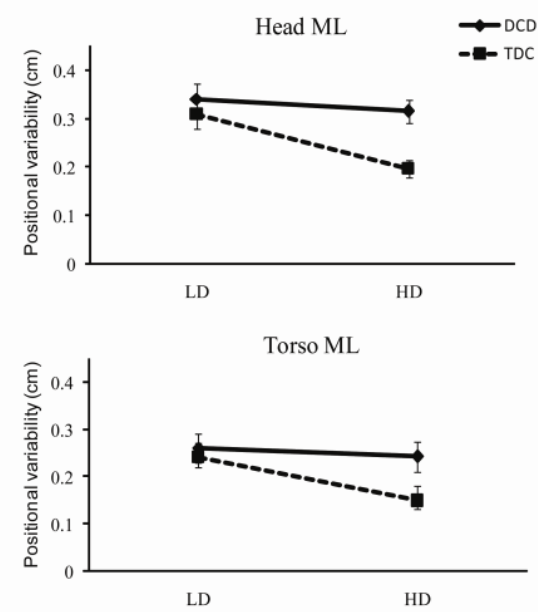

Figure 4. Positional variability of the head and torso in the ML axis.

\section{Discussion}

Riccio and Stoffregen [9] and Stoffregen et al. [10, 11] have proposed a functional integration between postural activity and a supra-postural task: Control of postural motion is not an aim in itself, but is valuable to the extent that it promotes success of other 
behavioral goals. Postural control is tuned to positively mediate performance of supra-postural tasks. Our experiments suggest that children with DCD seem less able to modulate their postural motion than their typically developing peers, when engaged in perceptual and cognitive tasks. It demonstrates that both the perception-action link, and what we propose as a cognition-action link are both degraded in children at risk for DCD. Studies that report the linkage between biomechanical responses (posture) and behavioral activity (perceptual or cognitive) provide support for an embodied interpretation of human performance, rather than the more traditional 'executive function' interpretation [12 ]. Children diagnosed with, or at risk for DCD are typically seen as 'clumsy'. This motor difficulty is not associated with a concomitant intellectual deficit, thus an 'online executive control' interpretation seems less feasible. The reliance on neurological descriptors, such as 'executive' or 'online control' add little to our insights about the motor behavior observed in children with DCD. Rather, the possible degrading of the perception-action or cognition-action link supports a more ecological interpretation of DCD. Sensitivity to the demands of both the environment and task would seem to be a functional explanation of the motor difficulties exhibited by these children. In conclusion, our experiments bolster the proposition that DCD is a perceptual motor deficit characterized by a diminished perception-action or cognition-action coupling when a motor response is linked to tasks that demand increased perceptual or cognitive effort.

\section{References}

[1] American Psychiatric Association. Diagnostic and statistical manual of mental disorders (4th ed., revised). Washington, DC: Author., 2000.

[2] R. H. Geuze. Static balance and developmental coordination disorder. Human Movement Science, 22, 527-548, 2003.

[3] E. P. Przysucha, and M. J. Taylor. Control of stance and developmental coordination disorder: The role of visual information. Adapted Physical Activity Quarterly, 21, 19-33, 2004.
[4] C. L. Tsai, S. K. Wu, and C. H. Huang. Static balance in children with developmental coordination disorder. Human Movement Science, 27, 142-153, 2008.

[5] R. H. Geuze, and H. Börger. Children who are clumsy: Five years later. Adapted Physical Activity Quarterly, 10,10-21, 1993.

[6] T. A. Stoffregen, M. A. Riley, P. Hove, C. T. Bonnet, and B. G. Bardy. Postural stabilization of perceptual but not cognitive performance. Journal of Motor Behavior 39, 2, 126-138, 2007.

[7] C.H. Chang, M. G. Wade, T. A. Stoffregen, C.Y. Hsu, and C.Y. Pan. Visual tasks and postural sway in children with and without Autism Spectrum Disorder. Research in Developmental Disabilities, 31, 1536$1542,2010$.

[8] M. A. Riley, A. A. Baker, J. M. Schmit, and E. Weaver. Effects of visual and auditory short term memory tasks on the spatiotemporal dynamics and variability of postural sway. Journal of Motor Behavior, 37, 311-324, 2005

[9] G. E. Riccio, and T. A. Stoffregen. Affordances as constraints on the control of stance. Human Movement Science, 7, 265-300, 1988.

[10] T. A. Stoffregen, R. J. Pagulayan, B. G. Bardy, and L. J. Hettinger. Modulating postural conrol to facilitate visual performance. Human Movement Science, 19, 203-220, 2000.

[11] T. A. Stoffregen, B. G. Bardy, C. T. Bonnet, P. Hove, and O. Oullier. Postural sway and the frequency of horizontal eye movements. Motor Control, 11, 86102, 2007.

[12] P. H. Wilson, and B. E. McKenzie. Information processing deficits associated with developmental coordination disorder: A meta-analysis of research findings. Journal of Child Psychology and Psychiatry, 39, 829-840, 1998. 